\title{
Síntesis y caracterización de materiales híbridos orgánico-inorgánicos de APS/PDMS
}

\author{
I. GARCÍA-PERULERO, S. MURCIA-MASCARÓS, J. RUBIO , J. L. OTEO \\ Instituto de Cerámica y Vidrio. CSIC. Camino de Valdelatas s/n. Madrid, España
}

\begin{abstract}
El uso de 3-aminopropiltrietoxi silano (APS) como precursor inorgánico en la síntesis de materiales híbridos, en sustitución de otros alcóxidos tradicionales como el tetraetilortosilicato (TEOS), abre la posibilidad de incorporar grupos amino reactivos en la red tridimensional. Estos grupos pueden ser utilizados para anclar diferentes compuestos orgánicos al material. Por otro lado, la rápida velocidad de hidrólisis del APS, así como su autocatálisis permite trabajar en condiciones de síntesis muy suaves, tales como medios no ácidos y temperatura ambiente, evitando así la descomposición de los compuestos orgánicos activos. El objetivo de este trabajo es la síntesis y caracterización de nanocomposites en los que los alcóxidos tradicionales han sido sustituidos por APS. Los grupos amino primarios incorporados a la red inorgánica y presentes en la superficie del material se determinan mediante reacciones con cromóforos específicos.
\end{abstract}

Palabras clave: APS, PDMS, método sol-gel, material híbrido, aminas superficiales.

\section{Synthesis and characterization of aps/pdms organic-inorganic hybrid materials}

The use of 3- aminopropyltriethoxy silane (APS) as inorganic precursor in the synthesis of hybrid materials in substitution of traditional alkoxides such as tetraethylorthosilicate (TEOS), opens up the possibility of incorporate reactive amine groups into the three-dimensional network. These groups can be used in order to link different organic compounds to the material. On the other hand, the short times of APS hydrolysis, allow to work under very soft conditions, such us non acid media and room temperature, hence avoiding the decomposition of the organic active compounds. The aim of this work is the synthesis and characterization of nanocomposites in which traditional alkoxides are replace by APS. Primary amine groups incorporated into the inorganic network and present on the material surface are determined by reaction with specific chromophores.

Keywords: APS, PDMS, sol-gel method, hybrid material, surface amines.

\section{INTRODUCCIÓN}

El 3-aminopropiltrietoxi silano (3-APS, $\gamma$-APS o APS) se viene usando desde hace décadas como agente acoplante para la organifilización de gran variedad de superficies, tales como fibras de vidrio (1), geles de sílice (2), cerámicas y óxidos inorgánicos en general (3). La formación de capas superficiales de APS, con grupos $\mathrm{NH}_{2}$ unidos covalentemente a la molécula, puede utilizarse para anclar multitud de compuestos, como polímeros (4), a estas superficies inorgánicas.

Por otra parte, el método sol-gel permite la síntesis de materiales híbridos orgánico-inorgánicos, cuyas propiedades pueden ser modificadas y seleccionadas mediante la elección de condiciones de síntesis y precursores adecuados (5). Es por esto que los geles de sílice modificados se han usado recientemente como soportes físicos para la retención o dosificación controlada de muchos tipos de compuestos, desde moléculas pequeñas como geraniol o mentol (6), heparina (7), hasta células completas tanto microbianas (8) como células complejas de plantas (9) y animales superiores (10), pasando por la incorporación de esteroides (11), antígenos (12) y multitud de enzimas y proteínas (13-15).
El empleo del APS como precursor en la síntesis de estos materiales híbridos (16) es más reciente y permite la incorporación de sus grupos amino primarios a la red tridimensional de una matriz esencialmente inorgánica, grupos funcionales que pueden ser posteriormente utilizados para el anclaje de diversas moléculas orgánicas, como pesticidas o compuestos antimicrobianos que quedarían unidos químicamente al material de soporte controlándose su liberación a través del control de la fortaleza del enlace químico presente.

En este trabajo, la síntesis de materiales híbridos se realizó utilizando APS, como formador de cadena, y polidimetilsiloxano (PDMS), un polímero lineal que confiere propiedades elastoméricas al material final (17). Mediante el método sol-gel el APS, un alkilalcóxido, sufre reacciones de hidrólisis (esquema 1) en sus tres cadenas hidrolizables y posteriores reacciones de autocondensación (esquema 2) y de condensación (esquema 3) con el PDMS, permaneciendo la cadena con el grupo amino funcional unida covalentemente a la matriz formada por enlaces Si-O-Si de los precursores. 


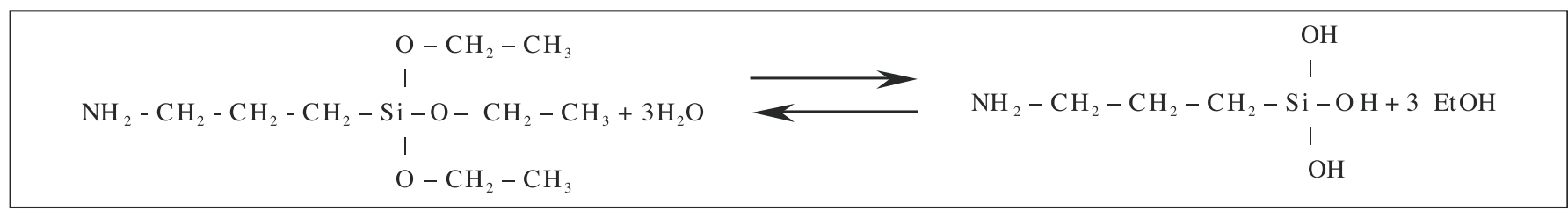

Esquema 1

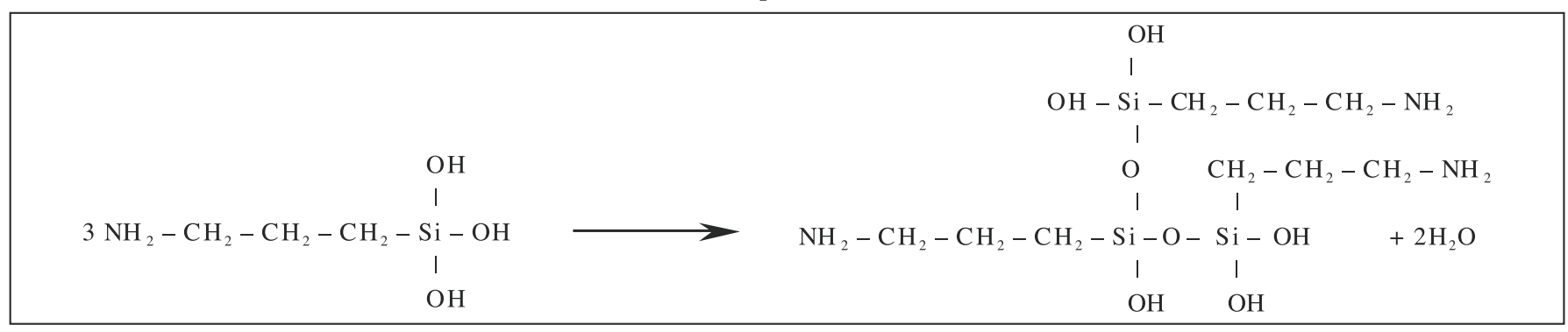

Esquema 2

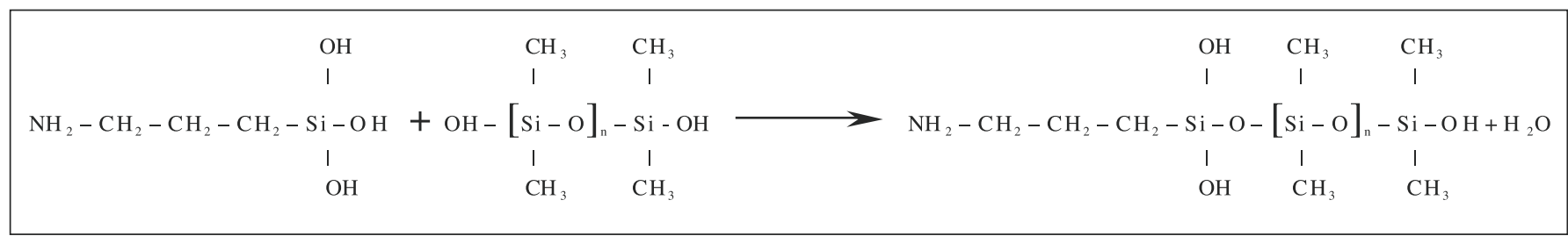

Esquema 3

Además, la presencia de los grupos amino primarios confiere un carácter básico al compuesto que es capaz de catalizar su propia hidrólisis (18) sin necesidad de añadir otros catalizadores ácidos o básicos que generen pHs extremos y utilizando la humedad atmosférica como aporte de agua (19). Así, las aminas primarias son capaces de protonarse captando grupos $\mathrm{H}_{3} \mathrm{O}^{+}$del agua y catalizar reacciones de hidrólisis por ataques nucleofílicos de los grupos $\mathrm{OH}^{-}$resultantes al enlace $\mathrm{Si}-\mathrm{OR}$. Esto permite trabajar en condiciones muy suaves de $\mathrm{pH}$ y temperatura, lo que facilitaría la adición de compuestos orgánicos muy sensibles, como por ejemplo las enzimas.

\section{EXPERIMENTAL}

Para la síntesis de los materiales se utilizaron los siguientes reactivos: APS (97\%) y PDMS de peso molecular PM (medio) = 550 de ABCR y Etanol absoluto (99.8\%) de MERCK como disolvente.

En este estudio se sintetizaron materiales en los que la cantidad de APS en relación a la de PDMS se fue aumentando desde un $20 \%$ en peso hasta un $60 \%$ (Tabla I).

La síntesis se realizó a temperatura ambiente añadiendo los reactivos (APS y PDMS) en un recipiente que contiene el disolvente (Etanol $n_{\mathrm{EtOH}} / \mathrm{n}_{\mathrm{APS}}=5$ ) y que se mantuvo en agitación mecánica a $600 \mathrm{rpm}$ durante 45 minutos. Una vez pasado el tiempo de reacción los soles de baja viscosidad se vierten en contenedores de plástico de $90 \mathrm{~mm}$ de diámetro (placas Petri), donde se dejan evaporar a temperatura ambiente y a humedad relativa del $35-40 \%$. Los soles van aumentando de viscosidad hasta que finalmente se obtienen sólidos del tamaño del contenedor, los cuales una vez secos (peso constante) se extraen del molde para su posterior caracterización.

La caracterización de los materiales se realizó utilizando diferentes técnicas. Para la determinación de las característi- cas microscópicas y de la microestructura se utilizó un microscopio electrónico de transmisión Hitachi H7100 a 125 Kv. Las muestras observadas corresponden a zonas de fractura.

Las medidas de los índices de opacidad se realizaron mediante un espectrofotómetro Minolta, modelo CM-2600d, con iluminante D65 (equivalente a luz diurna), 100\% de porcentaje de UV, ángulo de observador de $10^{\circ}$ y máscara de medida de 3 $\mathrm{mm}$ de diámetro. Estas medidas se realizan comparando las reflectancias de muestras y estándar (aire) sobre un fondo blanco (papel fotográfico de reflectancia aproximada del 89\%) y sobre un fondo negro (cartulina plástica) según la norma ISO 2471: 1998. Una vez obtenidos los espectros de reflectancia tanto para el estándar como para las muestras se determinó la utilización de los valores obtenidos con el modo SCE (componente especular excluída) debido a que los materiales presentan brillo.

El estudio de la superficie específica se realizó mediante un equipo de adsorción/desorción de nitrógeno modelo TriStar de Micromeritics.

Los sólidos se caracterizaron también mediante espectroscopia infrarroja por transformada de Fourier en pastillas, por dilución con KBr (MERCK), espectrómetro FTIR 1760X de Perkin Elmer.

El análisis elemental de C y S se realizó en el analizador LECO CS-200, mientras que el análisis de $\mathrm{N}$ y O fue realizado en un LECO EF-400.

La determinación de aminas primarias se hizo siguiendo el protocolo propuesto por Ngo (20) y modificado por Tyllianakis and col. (21) para la cuantificación de grupos amino funcionales en soportes sólidos. Los reactivos utilizados, descritos en dicha publicación, fueron 2-iminotiolano (ITL), ácido 5,5'ditiobis-(2-nitrobenzoico) DTNB, ditiotreitol (DTT), cloruro sódico $(\mathrm{NaCl}), \mathrm{EDTA}, \mathrm{Na}_{2} \mathrm{HPO}_{4}$ y $\mathrm{NaH}_{2} \mathrm{PO}_{4}$ de Sigma-Aldrich. Las medidas colorimétricas se realizaron en un espectrómetro UV-vis modelo Lambda 40 de Perkin Elmer. 


\section{RESULTADOS Y DISCUSIÓN.}

3.1 Influencia de la cantidad de APS en las características físicas, tiempos de gelificación y microestructura de los materiales

En este trabajo se han sintetizado materiales en los que la proporción APS/PDMS se varió desde 20/80 hasta 60/40 en peso. Los materiales obtenidos poseen características físicas diferentes, pudiendo formarse materiales transparentes con la consistencia de gomas o materiales duros y opacos. Un resumen de las muestras obtenidas puede verse en la Tabla I. Todas las muestras obtenidas gelificaron en forma de láminas de 90 mm de diámetro y de diferente espesor, que variaba también en función de la cantidad de APS, desde los $2 \mathrm{~mm}$ de la muestra 60/40, hasta los $4 \mathrm{~mm}$ de la muestra 20/80.

Los materiales con mayor proporción de APS presentan varias fases, ya que el material al $60 \%$ es blanco y opaco $(82.3 \%$ de índice de opacidad), probablemente debido a la formación de agregados ricos en el alcóxido. La cantidad de agregados disminuiría al disminuir la cantidad de alkilalcóxido, siendo prácticamente inapreciable en la muestra con $40 \%$ de APS. Los materiales con un $30 \%$ y $20 \%$ en peso de APS son totalmente transparentes (8.2 y $7.7 \%$ respectivamente) y homogéneos a simple vista.

Los tiempos de gelificación (tiempo en el que se obtienen geles húmedos, cuando al inclinar el recipiente que contiene el sol éste no fluye por acción de la gravedad) aumentan desde 10-12 horas hasta 170 horas al disminuir la cantidad de APS presente en la muestra. Esto es debido probablemente a la autocatálisis de las reacciones de hidrólisis y condensación producida por los grupos amino funcionales que permitiría la gelificación de materiales en tiempos cortos a partir de una cierta cantidad de APS.

En este tipo de reacciones donde la cantidad de agua viene determinada por la humedad atmosférica (sin adición de agua en la reacción) la velocidad de formación del gel está determinada fundamentalmente por la velocidad de la reacción de hidrólisis. Por lo tanto, a mayores concentraciones de APS el tiempo de gelificación sería menor indicando una hidrólisis más rápida debida a la mayor proporción de grupos amino reactivos disponibles.

La variación de los tiempos de gelificación en función de la proporción de alkilalcóxido puede verse en la Tabla I.

Las muestras obtenidas se estudiaron mediante microscopía electrónica de transmisión. Las micrografías de fractura

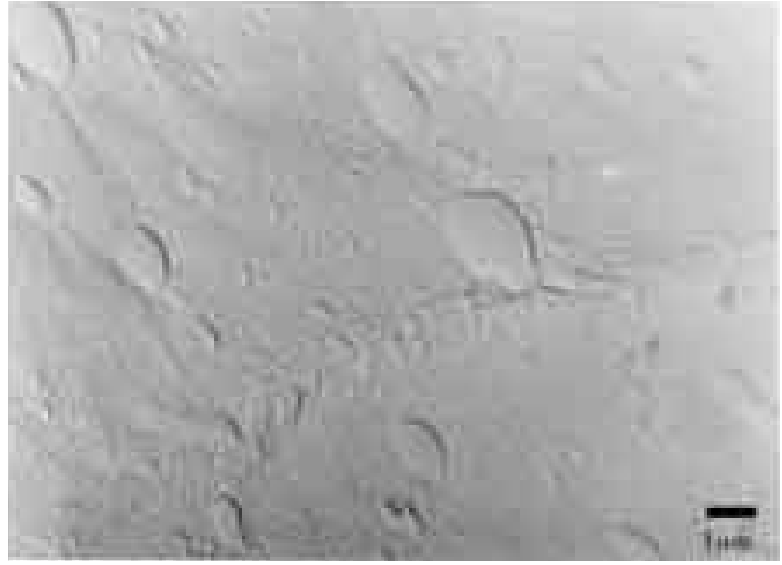

Figura 1. Imagen obtenida por microscopía electrónica de tranmisión de la muestra con $60 \%$ de APS. $(20 \mathrm{~A})$

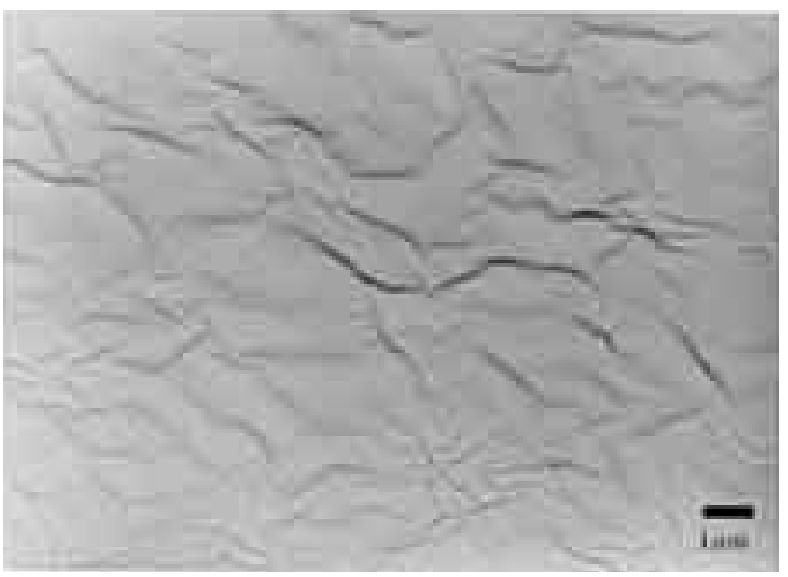

Figura 2. Imagen obtenida por microscopía electrónica de transimisión de la muestra con un $20 \%$ de APS. (20 A)

del material se muestran en las figuras 2 y 3 . La figura 1 corresponde al material con mayor contenido en APS en el que se observaban agregados globulares de APS rodeados de zonas enriquecidas en PDMS. Tanto la cantidad como el tamaño de estos agregados van disminuyendo a medida que disminuye la cantidad de APS, siendo inexistentes en las muestras de composición 20/80 (figura 2). Esto sugiere que las reacciones de autocondensación del APS (esquema 1) están favorecidas con respecto a las reacciones de policondensación APS-PDMS (esquema 2) cuando la cantidad de PDMS en el sistema es menor, produciéndose dos fases enriquecidas cada una de ellas en uno de los precursores. Las muestras con menor cantidad de

TABLA I. CANTIDADES DE APS Y PDMS EMPLEADAS EN LA PREPARACIÓN DE LAS MUESTRAS Y ALGUNAS CARACTERÍSTICAS FÍSICAS DE LOS MATERIALES FORMADOS.

\begin{tabular}{|c|c|c|c|c|c|c|c|c|}
\hline Muestra & $\begin{array}{c}\text { APS } \\
\text { (\% en peso) }\end{array}$ & $\begin{array}{c}\text { PDMS } \\
\text { (\% en peso) }\end{array}$ & $\begin{array}{c}\text { Índice de } \\
\text { opacidad }(\%) *\end{array}$ & $\begin{array}{c}\text { Propiedades } \\
\text { mecánicas } \\
\text { cuantitativas }\end{array}$ & $\begin{array}{l}\text { Espesor } \\
(\mathrm{mm})\end{array}$ & $\begin{array}{c}\text { \% C } \\
\text { (Teórico/ } \\
\text { Experimental) }\end{array}$ & \% Hidrólisis & $\begin{array}{c}\% \mathrm{~N} \\
\text { (Teórico/ } \\
\text { Experimental) }\end{array}$ \\
\hline 1 & 20 & 80 & 7.68 & Flexible, blando & 2.32 & $30.33 / 31.6$ & 78.56 & $1.82 / 3.21$ \\
\hline 2 & 30 & 70 & 8.23 & Flexible & 2.85 & $29.82 / 31.65$ & 74.77 & $2.75 / 2.65$ \\
\hline 4 & 50 & 50 & 28.64 & Rígido & 3.26 & $28.81 / 31.65$ & 72.95 & $4.62 / 5.32$ \\
\hline 5 & 60 & 40 & 82.33 & Rígido, duro & 4.05 & $28.30 / 31.2$ & 71.79 & $5.61 / 5.98$ \\
\hline
\end{tabular}

* Estándar transparente (aire): Índice de opacidad 7.5 
agregados son más transparentes, por lo tanto, la aparición de estos aglomerados en las muestras con mayor opacidad sugiere que la formación de una fase más rica en el alkilalcóxido es la causante de la falta de transparencia del material final.

Por otra parte, los estudios realizados mediante isotermas de adsorción/desorción de nitrógeno muestran xerogeles muy poco porosos, con superficies específicas BET muy pequeñas (inferiores a $0.5 \mathrm{~m}^{2} / \mathrm{g}$ ) aunque mayores para los materiales con mayor proporción de APS. Este ligero aumento del área superficial producido al aumentar la concentración del APS está relacionado con la disminución en los tiempos de gelificación producida por una hidrólisis más rápida que, como se dijo anteriormente, está favorecida por la presencia de mayor número de aminas primarias capaces de captar protones del medio. El tamaño medio de poro varía entre 37 y $15 \mathrm{~nm}$ aunque no depende de la cantidad de APS introducido.

\subsection{Análisis elemental.}

Los resultados experimentales del análisis elemental de carbono se muestran en la Tabla I. Se observa que la cantidad de $C$ experimental es en todos los casos, mayor que la calculada. En este tipo de materiales la cantidad de $\mathrm{C}$ tiene dos componentes, uno fijo procedente de los grupos metilo del PDMS y de los grupos aminopropilo del APS (ecuación 1), y otro componente variable que depende del grado de hidrólisis de los grupos etóxido del alquilalcóxido.

$$
\mathrm{C}_{\mathrm{T}}=\mathrm{M}_{\mathrm{PDMS}} * \mathrm{C}_{\mathrm{PDMS}}+\mathrm{M}_{\mathrm{APS}} * \mathrm{C}_{\mathrm{APS}}^{\mathrm{T}}
$$

$$
\begin{array}{ll}
\mathrm{C}_{\mathrm{T}}: & \begin{array}{l}
\text { Porcentaje de Carbono total esperado; } \\
\mathrm{M}_{\mathrm{PDMS}}:
\end{array} \\
& \begin{array}{l}
\text { masa PDMS; } \mathrm{C}_{\mathrm{PDMS}}: \text { Porcentaje de Carbono aporta- } \\
\text { do por el PDMS; }
\end{array} \\
\mathrm{M}_{\mathrm{APS}}: \quad \begin{array}{l}
\text { masa APS; } \mathrm{C}^{\mathrm{T}}{ }_{\mathrm{APS}}: \text { Porcentaje teórico de Carbono } \\
\text { aportado por el APS, considerando hidrólisis com- } \\
\text { pletas }
\end{array}
\end{array}
$$

La cantidad de C experimental también podría aumentar si parte del disolvente quedase ocluido en los poros del material, sin embargo, las muestras se han analizado tras un tiempo de secado prolongado (cuando los sólidos alcanzaban peso constante) y además la porosidad es muy pequeña, por lo que esta última contribución se puede considerar despreciable. Calculando el porcentaje de carbono aportado por el PDMS (que sólo depende de la cantidad de este y por lo tanto puede calcularse teóricamente) se puede calcular el porcentaje de carbono aportado por el APS (ecuación 2).

$$
\mathrm{C}^{\mathrm{R}}{ }_{\mathrm{APS}}=\mathrm{C}_{\mathrm{OBT}}-\mathrm{C}_{\mathrm{PDMS}}
$$

$\mathrm{C}^{\mathrm{R}}{ }_{\mathrm{APS}}$ : Porcentaje real de Carbono aportado por el APS

$\mathrm{C}_{\mathrm{PDMS}}$ : Porcentaje real de Carbono aportado por el PDMS, coincide con el porcentaje teórico.

$\mathrm{C}_{\mathrm{OBT}}$ : Porcentaje de Carbono obtenido experimentalmente

Para hacer un cálculo del porcentaje de APS hidrolizado en cada caso basta con relacionar los porcentajes de carbono esperados con el carbono obtenido experimentalmente, mediante sencillas ecuaciones aritméticas por las que se llega a la relación (ecuación 3).

$$
\mathrm{H}=\frac{\mathrm{C}_{\mathrm{OBT}}-\mathrm{C}_{\mathrm{PDM} \mathrm{S}}-\mathrm{C}_{\mathrm{APS}-\mathrm{EtOH}}}{\mathrm{C}_{\mathrm{APS}-\mathrm{OH}}-\mathrm{C}_{\mathrm{APS}}} * 100
$$

H: $\quad$ Porcentaje de APS hidrolizado;

$\mathrm{C}_{\mathrm{OBT}}$ : Porcentaje de Carbono obtenido experimentalmente;

$\mathrm{C}_{\mathrm{PDMS}}$ : Porcentaje de Carbono teórico aportado por el PDMS;

$\mathrm{C}_{\text {APS-EtOH }}$ : Porcentaje de Carbono teórico aportado por el APS sin hidrolizar;

$\mathrm{C}_{\mathrm{APS}-\mathrm{OH}}$ : Porcentaje de Carbono teórico aportado por el APS hidrolizado.

Estos cálculos indican que, en todos los casos, la hidrólisis no es completa, estableciéndose un equilibrio en la reacción, que alcanza un máximo del 78\% del APS presente. Este porcentaje de APS hidrolizado, depende de la cantidad de dicho compuesto, siendo mayor cuanto mayor sea esta cantidad (Tabla I). Esto vendría a confirmar que la presencia de aminas primarias genera una catálisis básica que activa la reacción de hidrólisis del compuesto.

Por otro lado, la cantidad de $\mathrm{N}$ teórico, considerando la hidrólisis incompleta del APS previamente calculada, se ajusta adecuadamente a la cantidad de $\mathrm{N}$ obtenida experimentalmente (Tabla I). Se observa una mayor discrepancia entre ambos valores en muestras con menor cantidad de APS (20\%), debido a la imprecisión del equipo para pequeñas cantidades de nitrógeno, cuando la muestra contiene oxígeno.

Todo esto indicaría que, en las condiciones experimentales propuestas (ausencia de catalizadores, temperatura ambiente y aporte de agua procedente de la humedad atmosférica - 35$40 \%$ ) el APS es capaz de catalizar su propia reacción de hidrólisis muy lentamente (tiempos de gelificación muy altos) hasta establecer un equilibrio entre el etanol formado en dicha reacción y el etanol añadido como disolvente de los reactivos, que permitiría alcanzar porcentajes de hidrólisis que en ningún caso superan el $80 \%$. Esto confirmaría los datos observados por espectroscopia, que se discutirán a continuación.

\subsection{Estudio de los sólidos obtenidos mediante espectrosco- pia infrarroja por transformada de Fourier (FTIR).}

Los materiales obtenidos presentan espectros de IR propios de materiales con alto contenido en sílice amorfa, con las diversas variaciones debidas a la inclusión de grupos orgánicos en la red (22). Todas las zonas del espectro aportan datos esenciales para la determinación de la estructura del material final y por ello la espectroscopia infrarroja ha sido considerada una herramienta muy útil para el estudio de la estructura del APS, por lo que sus bandas están bien descritas en la bibliografía (23) y pueden ser comparadas con las bandas características del TEOS o de otros alcóxidos $(24,25)$. 
La zona entre 4000 y $2000 \mathrm{~cm}^{-1}$ presenta las bandas de vibraciones de tensión características de los grupos $\mathrm{OH}$, tanto del agua adsorbida por el $\mathrm{KBr}$ como de los grupos silanoles. Esta banda, de gran intensidad solapa con las bandas de tensión asimétrica y simétrica de las aminas primarias del APS, que aparecen entre 3500 y $3300 \mathrm{~cm}^{-1}$ como un doblete característico, por lo que no es útil para determinar la estructura del APS en el material. En $2964 \mathrm{~cm}^{-1}$ aparecen las vibraciones de tensión asimétrica del $\mathrm{CH}_{3}$ correspondiente al PDMS. Con un número de onda algo menor $\left(2645 \mathrm{~cm}^{-1}\right)$ y de menor intensidad aparecen las bandas de vibración de tensión asimétrica de los $\mathrm{CH}_{2}$ presentes en la cadena no hidrolizable del APS. Estas bandas no presentan cambios al sucederse las reacciones de hidrólisis y condensación, por lo que solo indican que el APS está incorporado en el material.

En la zona comprendida entre 900 y $400 \mathrm{~cm}^{-1}$ (figura 3), la banda a $846 \mathrm{~cm}^{-1}$, correspondiente a las vibraciones de los enlaces $\mathrm{SiO}^{3-}$, corrobora la presencia de grupos silanoles provenientes del APS hidrolizado.

La zona de mayor importancia por los datos que aporta a la estructura del material, es la comprendida entre 1750 y $500 \mathrm{~cm}^{-1}$ (figura 3). En esta zona aparecen todas las bandas de tensión de los enlaces $\mathrm{Si}-\mathrm{O}$ - Si. Estas bandas, presentes ya en el PDMS, aumentan en anchura y se desplazan desde los 1088 $\mathrm{cm}^{-1}\left(v_{\mathrm{a}}\right.$ en el PDMS) y $1034 \mathrm{~cm}^{-1}\left(v_{\mathrm{s}}\right.$ en el PDMS) hasta $1098 \mathrm{y}$ $1018 \mathrm{~cm}^{-1}$ respectivamente, indicando que se han producido reacciones de condensación con el APS. También en esta región aparecen las bandas de los grupos $\mathrm{Si}-\mathrm{OH}$ aproximadamente en $920 \mathrm{~cm}^{-1}$, indicando la presencia de silanoles libres, tanto del PDMS como del APS. La disminución de la intensidad de esta banda al disminuir la cantidad de APS indica que la mayor parte de los silanoles terminales proceden del compuesto hidrolizado.

Otra información importante se obtiene al examinar las bandas correspondientes al etanol y a los grupos etoxi del alkilalcóxido. La ausencia de la banda de etanol libre a $880 \mathrm{~cm}^{-1}$ indica que todo el alcohol, tanto el añadido como disolvente como el generado en la hidrólisis del compuesto, se ha evaporado durante los procesos de gelificación y secado. Por otra parte, la presencia de bandas de pequeña intensidad correspondientes a los grupos etoxi del APS indican que el reactivo no se ha hidrolizado completamente, confirmando los datos obtenidos del análisis elemental de carbono. Estas bandas, a $1386 \mathrm{~cm}^{-1}$, $\delta_{\mathrm{s}} \mathrm{C}-\mathrm{H}$ en $\mathrm{CH}_{3}$ del etoxi del APS, y a $1194 \mathrm{~cm}^{-1} \rho_{\mathrm{r}} \mathrm{CH}_{3}$ (rocking) del grupo etoxi del APS, confirman la presencia de APS en la red, ya que disminuyen su intensidad al disminuir la cantidad de APS incorporado en el gel.

Las bandas de deformación de los grupos amino aparecen también en esta zona espectral, entre 1650 y $1300 \mathrm{~cm}^{-1}$, por lo que es en esta región donde se pueden apreciar mejor los cambios que se producen al introducir APS en el material. La ubicación de estas bandas y su relación con la estructura que adopta el APS al interaccionar consigo mismo o con otros materiales ha sido muy discutida. Desde la teoría del anillo pentacoordinado o del zwitterion de Plueddemann (26), en la que el nitrógeno interacciona con el átomo de silicio o con el grupo $\mathrm{OH}$ de uno de los silanoles respectivamente, hasta los estudios más recientes $(27,28)$ que apuestan por la formación de puentes de hidrógeno entre el grupo amino y grupos $\mathrm{Si}-\mathrm{OH}$ propios del compuesto o del material con el que reacciona, han sido muchos los autores que han tratado de explicar el desplazamiento desde $1610 \mathrm{~cm}^{-1}$ hasta $1582 \mathrm{~cm}^{-1}$ que sufre

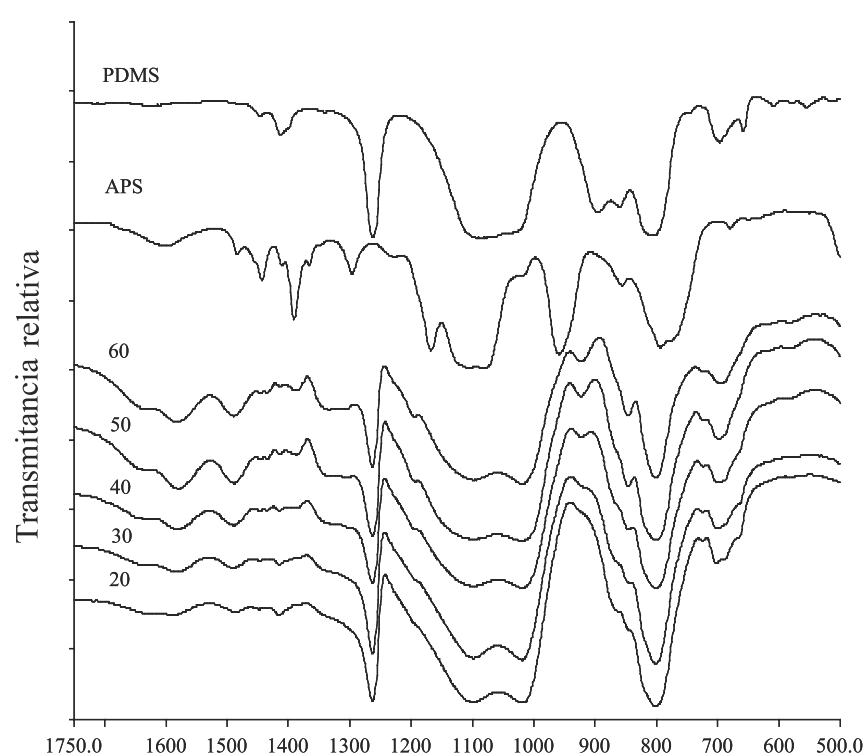

Número de onda $/ \mathrm{cm}^{-1}$

Figura 3. Espectros IR de las muestras sólidas en $\mathrm{KBr}$, en función de la cantidad de APS y de los reactivos iniciales (APS y PDMS)

la banda de flexión asimétrica del grupo amino primario, asî como el desplazamiento o aparición de otras bandas como las de $1630 \mathrm{~cm}^{-1} \mathrm{o}$ la de $1332 \mathrm{~cm}^{-1}$ que algunos autores asignan a la bicarbonatación del grupo $\mathrm{NH}_{2}$ producida por el $\mathrm{CO}_{2}$ del medio (29). Esta estructura depende tanto de las concentraciones de APS como de las condiciones de preparación del material ( $\mathrm{pH}$, atmósfera de secado, existencia de tratamiento térmico posterior, etc.).

En este trabajo se ha observado que las bandas correspondientes a las vibraciones de flexión de los grupos amino aumentan en intensidad al aumentar la concentración del APS, indicando la incorporación de éste a la estructura de la matriz inorgánica. Sin embargo, los estudios de espectroscopia y de análisis elemental de muestras calentadas en estufa a $120^{\circ} \mathrm{C}$, como se propone en el trabajo de Culler and col. (30), no son suficientes para descartar la aparición de grupos bicarbonato o de puentes de hidrógeno que impliquen a las aminas y que disminuirían su reactividad. Por ello es importante realizar otros ensayos que permitan determinar la cantidad de aminas primarias reactivas en el material final. El método propuesto en este trabajo es un método colorimétrico cuyos resultados se muestran a continuación.

\subsection{Determinación colorimétrica de aminas primarias.}

El empleo de APS como agente acoplante sobre superficies silíceas ha sido ampliamente estudiado $(1,26)$. Existen numerosos estudios que tratan de caracterizar la estructura que adopta el compuesto al reaccionar con los grupos $\mathrm{Si}-\mathrm{OH}$ de los materiales que se recubren. Estos estudios muestran la existencia de una gran variedad de estructuras que incluyen desde enlaces 


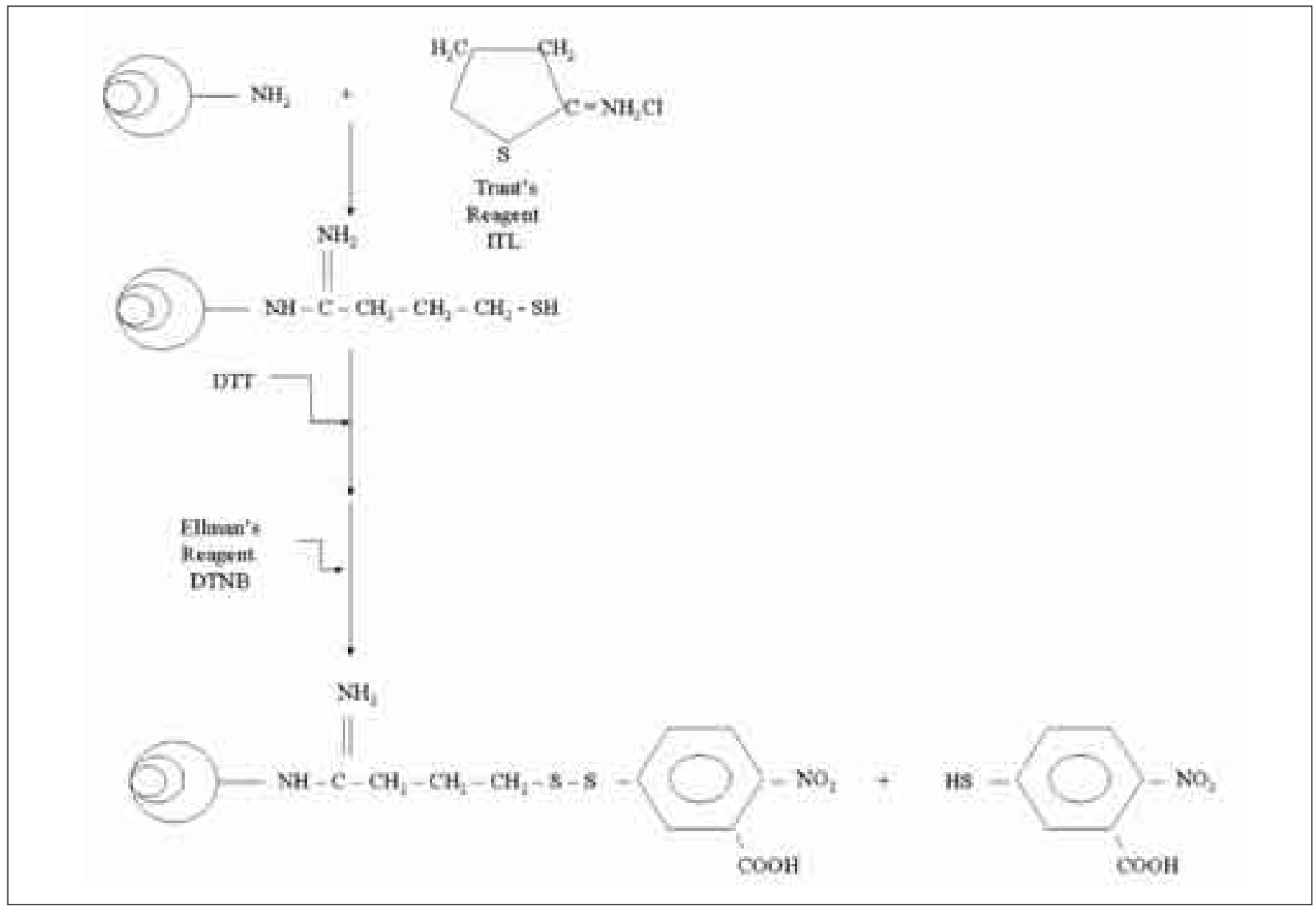

Figura 4. Determinación colorimétrica de aminas primarias. Esquema de la reacción de Ngo.

de condensación de los grupos $\mathrm{Si}-\mathrm{OH}$ hidrolizados del APS hasta puentes de $\mathrm{H}$ formados entre el material y los grupos amino del compuesto. Es por esto que la determinación de la cantidad de grupos amino primarios reactivos contenidos en el material sólido es fundamental para su caracterización, aún más si estos grupos amino van a ser utilizados como grupos activos para el anclaje de otras moléculas. Como se mencionó anteriormente, la espectroscopia IR realizada en pastillas de $\mathrm{KBr}$ produce solapamientos de las bandas características de los grupos aminos con las bandas del agua adsorbida por el $\mathrm{KBr}$, por lo que las técnicas de espectroscopia IR no son válidas para la detección y cuantificación de dichos grupos. Es por esto que se optó por las técnicas de cuantificación colorimétrica para esta caracterización.

Existen numerosas técnicas colorimétricas de determinación de aminas, pero es la propuesta por Ngo la que permite de modo más sencillo la cuantificación de estos grupos en compuestos anclados a medios sólidos. Este método se basa en hacer reaccionar soportes sólidos que contienen grupos amino con un reactivo con alta especificidad por dichos grupos (ITL) y posteriormente con un reactivo con especificidad para los grupos tiol (DTNB). La reacción del ITL con los grupos amino resulta en la formación de enlaces amidina cargados positivamente y por tanto, en la introducción de grupos sulfhidrilo a través de la ruptura del anillo de tiolano. Los sulfhidrilos son entonces valorados usando el reactivo de Ellman (DTNB). El esquema de la reacción completa se muestra en la figura 4.

La cuantificación de los grupos amino primarios se rea- liza haciendo reaccionar mol a mol dichos grupos con una disolución que contiene ITL. El material así tratado se lava posteriormente con una segunda disolución del compuesto DTNB, compuesto que al reaccionar con el ITL se hidroliza liberando en la disolución $\mathrm{TNB}^{2-}$. La medida de los cambios en la absorbancia a $\lambda=412 \mathrm{~nm}$ (longitud de onda en la que esta especie presenta el máximo de absorción) de la disolución resultante permite conocer de forma indirecta la cantidad de grupos amino que han reaccionado con el ITL y su cuantificación mediante la ley de Lambert-Beer, ya que el coeficiente de extinción molar del $\mathrm{TNB}^{2-}$ es conocido.

Este método se aplicó en la cuantificación de aminas superficiales de las muestras obtenidas. Los resultados obtenidos se muestran en la figura 5. Se puede observar que para muestras con cantidades de APS inferiores al $40 \%$ la concentración de aminas superficiales se ajusta a una recta que pasa por el origen y que alcanza un valor máximo del $2.4 \%$ (muestra con $40 \%$ de APS), incrementándose linealmente con el aumento de la cantidad de APS en la muestra. Sin embargo a partir de dicha cantidad no se cumple la misma ecuación lineal. Esto se puede traducir en un ajuste a una curva logarítmica que pasa por el origen y tiende a un valor límite marcado por la formación de aglomerados. Este resultado estaría de acuerdo con los datos previamente discutidos, ya que las muestras con mayor cantidad de APS, formarían más agregados de dicho compuesto, por lo que tendrían menor cantidad de aminas superficiales.

Otro hecho importante que afectaría a la concentración de aminas reactivas es su tendencia a formar puentes de hidróge- 


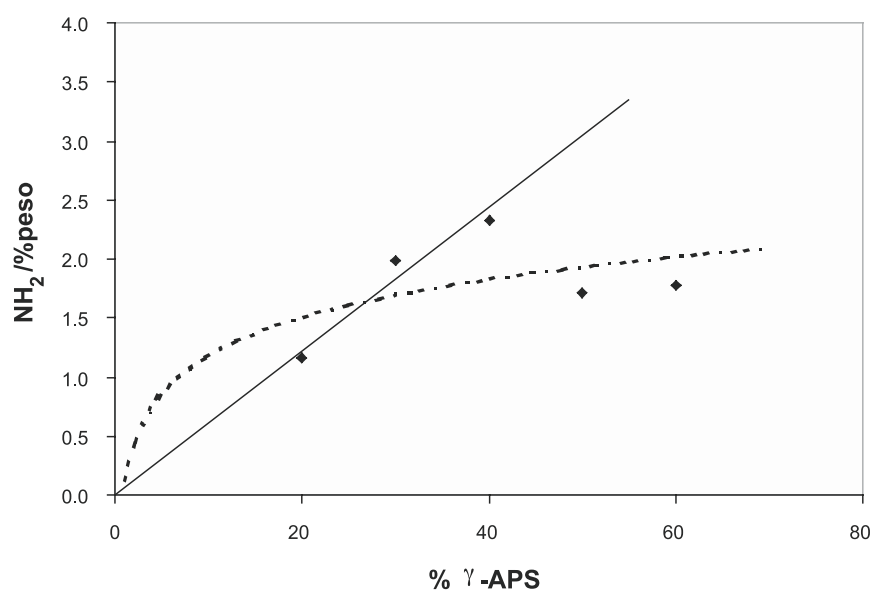

Figura 5. Variación de la concentración de aminas primarias en la superficie de las muestras con diferente contenido en APS.

no o a bicarbonatarse cuando se secan en atmósfera de aire, como demuestran los resultados discutidos previamente sobre el desplazamiento de las bandas del espectro de IR debidas a las tensiones de flexión de dichos grupos amino primarios.

Por lo tanto, a pesar de los interesantes resultados obtenidos con el método colorimétrico, no es posible establecer una relación entre la cantidad de aminas primarias reactivas y el porcentaje de nitrógeno de las muestras ya que los datos obtenidos solo son válidos para las aminas primarias en superficie. Las diferencias observadas entre los porcentajes de nitrógeno totales presentes en cada muestra y los porcentajes de aminas primarias pueden deberse a varias causas. La primera de ellas sería la formación de agregados de APS no superficiales, que impedirían la reacción de todos los grupos amino disponibles con el ITL. La segunda sería la formación de enlaces entre las aminas y el $\mathrm{CO}_{2}$ atmosférico (bicarbonatación), así como la posible formación de puentes de hidrógeno inter- o intracatenarios generarían porcentajes de aminas secundarias no reactivas. Así, la muestra con un $40 \%$ de APS presenta una cantidad total de nitrógeno del $3.6 \%$, mientras que la cantidad de aminas detectadas mediante el método colorimétrico sólo alcanza el 2.4\% como se comentó anteriormente, consiguiéndose por lo tanto un $66.7 \%$ de grupos amino reactivos disponibles. Por esto, es posible afirmar que el método empleado para la síntesis de estos materiales proporciona cantidades importantes de aminas primarias disponibles para el anclaje de otras moléculas.

\section{CONCLUSIONES}

En este estudio se ha establecido una metodología de preparación de materiales híbridos orgánico-inorgánicos que poseen en superficie grupos amino reactivos. Para ello se ha empleado un alcóxido amínico, el 3-aminopropiltrietoxi silano, capaz de formar compuestos homogéneos y transparentes con PDMS mediante un proceso sol-gel en condiciones muy suaves. Así se obtienen sólidos de espesor, transparencia y dureza variables en función de su utilidad.

La caracterización químico física de estos materiales permite establecer que la hidrólisis parcial y posterior condensación del APS da lugar a enlaces Si-O-Si en una red inorgánica a la que quedan unidos covalentemente los grupos amino pri- marios que mantienen su capacidad de reaccionar posteriormente con otras especies. En particular, empleando el método colorimétrico de $\mathrm{Ngo}$, se ha podido determinar que es posible obtener hasta un $2.4 \%$ en peso de grupos activos $\mathrm{NH}_{2}$ en la superficie del producto final, lo cual supone un porcentaje del $66.7 \%$ de aminas primarias en superficie disponibles para el anclaje de otros compuestos.

Estos resultados demuestran la posibilidad de obtener soportes sólidos con grupos reactivos de naturaleza similar a la de las moléculas biológicas, lo que facilitaría su anclaje y posterior dosificación; moléculas tales como las enzimas u otros compustos bioactivos, que se desnaturalizan fácilmente en condiciones de reacción agresivas, por lo que el método de preparación aquí descrito podría ser el adecuado.

Por último, se ha establecido que el material más homogéneo, con mayor cantidad de grupos amino superficiales y con mejores propiedades ópticas y mecánicas, es el que contiene una cantidad de APS en torno al $40 \%$.

\section{AGRADECIMIENTOS}

IGP agradece al CSIC la concesión de una beca I3P para la realización de este trabajo

\section{BIBLIOGRAFIA}

1. M.C. Bautista Sanz. "Estudio de fibras de vidrio sílice-alúmina para su utilización en materiales compuestos". Tesis Doctoral. Madrid (1994).

2. K.C. Vrancken, P. Van der Voort, K. Possemiers and E.F. Vansant. "Surface and Structural Properties of Silica Gel in the Modification with $\gamma$-Aminoprop yltriethoxysilane" J. Colloid and Interface Science 174, 86-91 (1995).

3. C.W. Chu, D.P. Kirby and P.D. Murphy. "Interactions of aminosilane with alumina and silica substrates deposited from nonaqueous and aqueous media" J. Adhesion Sci. Technol, Vol 7 (5), 417-433 (1993)

4. H.G. Linde. "Polyamic Acid Interactions with Aminopropylsilane Surface Conditoners on Metal" Appl. Polym. Sci. 40, 613-622 (1990)

5. J.D. Mackenzie and E.P. Bescher. "Structures, Properties and Potential Applications of Ormosils" J. Sol-Gel Sci. And Technol 13, 371-377 (1998).

6. C. Carturan; E. Pagani, R. Campostrini and R. Ceccato. "Hybrid Gels as Host Matrices of Perfumed Essences" J. Sol-Gel Sci. And Technol 8, 1115-1119 (1997).

7. M.S. Ahola, E. S. Säilynoja, M. H. Raitavuo, M. M. Vaahtio, J. I. Salonen and A. U. O. Yli-Urpo. "In vitro release of heparin from silica xerogels" Biomaterials Vol 22 (15), 2163-2170 (2001).

8. T. Brányik, G. Kuncová, J. Páca and K. Demnerová. “Encapsulation of Microbial Cells into Silica Gel" J. Sol-Gel Sci. and Technol. 13, 283-287 (1998).

9. G. Carturan, R. Dal Monte, G. Pressi, S. Secondi and P. Verza. "Production of Valuable Drugs from Plant Cells Immobilized by Hybrid Sol-Gel $\mathrm{SiO}_{2}$ " J. Sol-Gel Sci. and Technol. 13, 273-276 (1998).

10. E. J. A. Pope, K. Braun and C. M. Peterson. “Bioartificial Organs I: Silica Gel Encapsulated Pancreatic Islets for the Treatment of Diabetes Mellitus" J. SolGel Sci. and Technol. 8, 635-639 (1997).

11. L. Siemska, M. Ferguson, T. W. Zerda and E. Couch. “Diffusion of Steroids in Porous Sol-Gel Glass: Application in Slow Drug Delivery" J. Sol-Gel Sci. and Technol. 8, 1105-1109 (1997).

12. C. Roux, J. Livage, K. Farhati and L. Monjour. “Antibody-Antigen Reactions in Porous Sol-Gel Matrices" J. Sol-Gel Sci. and Technol. 8, 663-666 (1997)

13. L. M. Ellerby, C.R. Nishida, F. Nishida, S. A. Yamaka, B. Dunn, J. Selverstone Valentine and J. I. Zink. "Encapsulation of Proteins in Transparent Porous Silicate Glasses Prepared by the Sol-Gel Method" Science 255, 1113-1115 (1992).

14. G. Kuncová and M. Sivel. "Lipase Inmobilized in Organic-Inorganic Matrices" J. Sol-Gel Sci. and Technol. 8, 667-671 (1997).

15. B. C. Dave, J. M. Miller, B. Dunn, J. S. Valentine and J. I. Zink. “Encapsulation of Proteins in Bulk and Thin Film Sol-Gel Matrices" J. Sol-Gel Sci. and Technol. 8, 629 (1997). 
16. Y. Han, J. Lin and H. Zhang. "Photoluminescence of organic-inorganic hybrid $\mathrm{SiO}_{2}$ xerogels" Materials Letters 54, 389-396 (2002).

17. O. Foussaier, M. Menetrier, J.J. Videau and E. Duguet. "Polydimethylsiloxanebased ORMOSIL microstructure: correlation with compressive behavior" Matterials Letters 42, 305-310 (2000)

18. F. Beari, M. Brand, P. Jenker; R. Lehnert, H.J. Metternisch, J. Monkiewicz and H.W. Siesler. "Organofunctional alkoxysilanes in dilute aqueous solution: new accounts on the dynamic structural mutability" J. Organometallic Chemistry 625, 208-216 (2001).

19. K.C. Vrancken, P. Van der Voort, I. Gillis-D'Hamers and E.F. Vasant "Influence of Water in the Reaction of $\gamma$-Aminopropyltriethoxysilane with Silica Gel” J. Chem. Soc. Faraday Trans. 88 (21), 3197-3200 (1992).

20. T.T. Ngo. "Colorimetric Determination of Reactive Amino Groups of a Solid Support Using Traut's and Ellman's Reagents" Applied Biochem. and Biotech. 13, 213-219 (1986).

21. P. Tyllianakis, S.E. Kakabakos, G.P. Evangelatos and D.S. Ithakissios "Colorimetric Determination of Reactive Primary Amino Groups of Macroand Microsolid Supports" Applied Biochem. and Biotech. 38, 15-25 (1993).

22. R.T. Conley. En: "Infrared Spectroscopy". Ed Alhambra, Spain (1972).

23. H. Ishida and Y. Suzuki. "Hydrolysis and condensation of aminosilane coupling agents in high concentration aqueous solutions: a simulation of silane interphase" En: “Compos. Interfaces, Proc. Int. Conf.”, $1^{\text {st }}, 317-327$. Ed.
North Holland: New York. (1986).

24. F. Rubio, J. Rubio and J.L. Oteo. "A FT-IR Study of the hydrolysis of tetraethylorthosilicate (TEOS)" Spectroscopy Letters 31 (1), 199-219 (1998)

25. D.L. Ou and A.B. Seddon. "Near- and mid-infrared spectroscopy of sol-gel derived ormosils: vinyl and phenyl silicates" J. Non-Crystalline Solids 210, 187-203 (1997).

26. E.P. Plueddemann. En: "Silane Coupling Agents". Ed Plenum Press. Nueva York. 1982.

27. I. Shimizu, H. Okabayashi, K. Taga, E. Nishio and C.J. O'Connor. “Diffuse reflectance infrared Fourier transform spectral study of the thermal and adsorbed-water effects of a 3-aminopropyltriethoxysilane layer modified onto the surface of silica gel" Vibrational Spectroscopy 14, 113-123 (1997).

28. A.A. Golub, A.I. Zubenko and B.Z. Zhmud. " $\gamma$-APTS Modified Silica Gels: The Structure of the Surface Layer" J. Colloid and Interface Science 179, 482 487 (1996)

29. S. Naviroj, J.L. Koenig, H. Ishida. "Molecular Structure of an Aminosilane Coupling Agent as Influenced by Carbon Dioxide in Air, pH, and Drying Conditions" J. Macromol. Sci. - Phys, B22 (2), 291-304 (1983).

30. S.R. Culler. "Analytical and Spectroscopic Investigation of the interaction of $\mathrm{CO}_{2}$ with Amine Functional Silane Coupling Agents on Glass Fibers" J. Col. Int. Sci. 96 (1), 69-79 (1983).

Recibido: 04.08.03

Aceptado: 19.11 .03
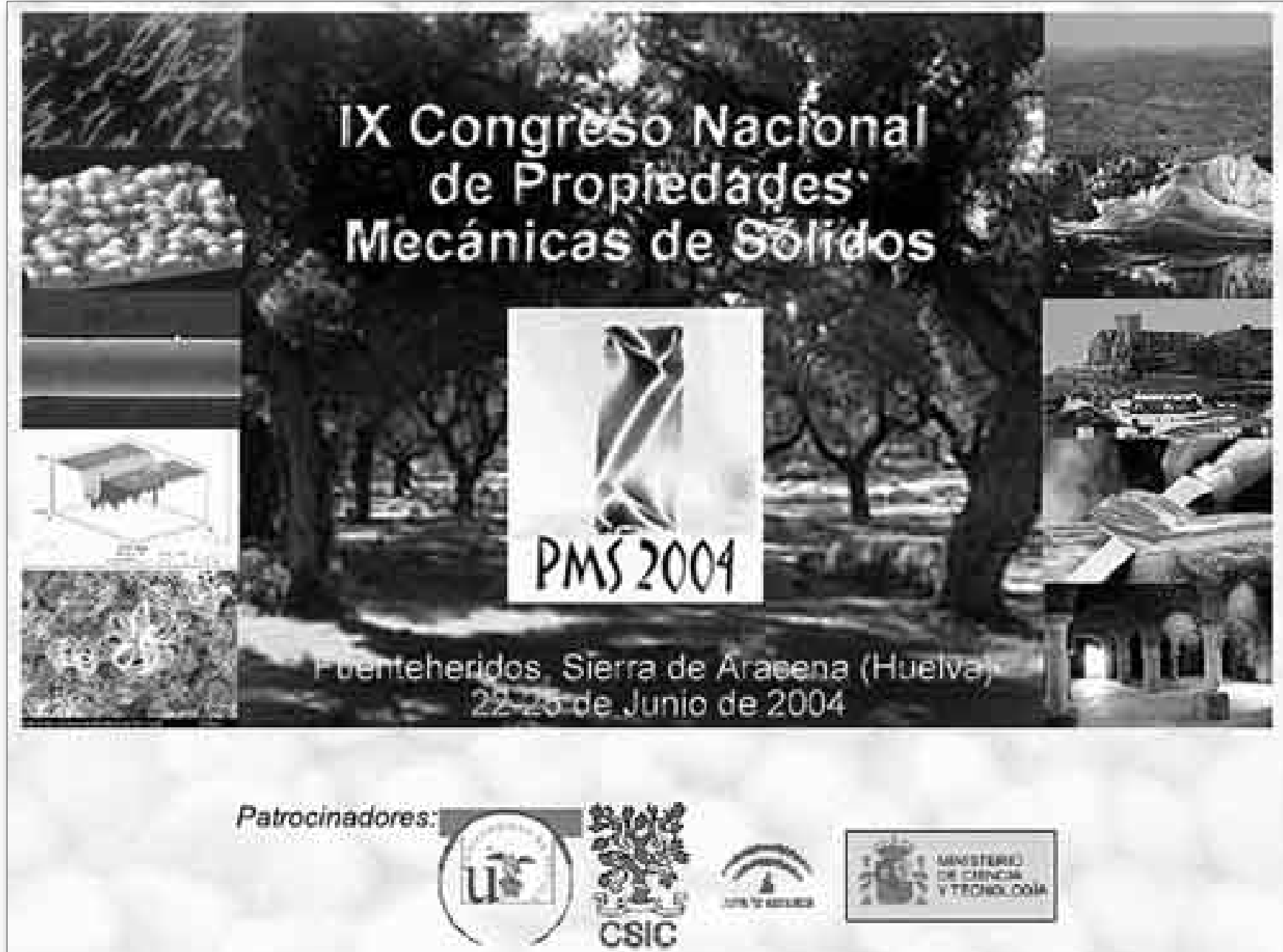

www.us.es / cnpms2004/ 